\title{
Protecting our children
}

\section{Rob Cushman, MD, FRCPC, ${ }^{1}$ Katherine Robertson-Palmer, $R N, M S c N^{2}$}

At a time when public health is pressing governments to protect our children from tobacco, the Canadian Journal of Public Health provides timely and useful information about the impact of the Ontario Tobacco Control Act (OTCA) in schools. The article by Northrup et al. ${ }^{1}$ reports on the perceptions of school administrators following the smoking ban on school property since the OTCA was passed. The companion article by Ashley et al. ${ }^{2}$ discusses issues and challenges arising from implementation and enforcement of the OTCA.

What we are witnessing is the progression of a public health program from education and promotion to legislation and ultimately enforcement. History tells us that this evolution is the only way to push compliance beyond a sub-optimal plateau, whether it be for seat-belts, tobacco, or any other intervention.

Before addressing tobacco control in our schools, it is worth reviewing and updating what we know about tobacco and youth, so as to define the problem and the importance of enforcement as a strategy.

Nationally, the prevalence of smoking among teens aged $15-19$ is 29\%. ${ }^{3}$ Due largely to 1994 federal tax reductions, teenage smoking is on an alarming increase. Students in Eastern Ontario increased their rate of smoking from $22 \%$ in 1993 to $29 \%$ in $1997 .{ }^{4}$ The vast majority of smokers start smoking in their very early to middle adolescent years and adult smoking patterns are generally determined between the ages of 16 and 18 years. ${ }^{3}$ The addiction to tobacco, once established, is ironclad. Adolescent smokers acknowledge their addiction to nicotine and want to quit; more than half of teens who are currently smoking have tried to quit for at least one week. ${ }^{3}$ However, only $2-3 \%$ of teens who initially

Regional Municipality of Ottawa-Carleton Health Department

1. Medical Officer of Health

2. Public Health Nurse, Tobacco Use Prevention Program, Adult Health Division made a quit attempt are successful for a year or two. ${ }^{5}$ Non-smoking youth are also vulnerable whether through involuntary exposure to highly toxic substances or through ongoing pressure to take up smoking.

Schools are obviously a key battlefield where the war on tobacco for the next generation of smokers will be won or lost. It is in this general environment amongst their friends and peers that kids will make and cement the most important individual health decision of their lives. This is why it is so important to review existing national school policies and learn from Ontario's experience from the Tobacco Control Act.

A 1995 Health Canada cross-province analysis demonstrated almost all (97\%) elementary and secondary schools had some type of policy, although the extent of the restrictions varied considerably. ${ }^{6}$ Prior to legislation, the extent to which schools provided a non-smoking policy varied widely among, and within, Ontario municipalities. Some schools prohibited students from smoking at school, while teachers and others were allowed to smoke in designated areas. With implementation of the OTCA, students no longer receive double messages at school about smoking.

\section{Ontario Tobacco Control Act, 1994}

The Ontario Tobacco Control Act (Bill 119) was passed in 1994 and, at the time, was considered the most comprehensive legislation in North America. The sections of the Act pertaining to youth were designed to reduce their smoking and protect them from environmental tobacco smoke. First, the Act reduces youth access to tobacco products by restricting the selling and supplying of tobacco to youth under 19, banning vending machines that sell or dispense tobacco and banning "kiddie packs" (packs with less than 20 cigarettes) or the selling of single cigarettes. Second, under section 9 of the Act, smoking is prohibited or restricted in a variety of places, including schools as defined in the Education Act. It bans smoking in all school buildings and on school properties at all times. It applies equally to staff, students and persons who use and visit school property. Schools are encouraged to extend this ban to outings and school buses through school policy.

The survey by Northrup et al. indicates that the OTCA is perceived as an acceptable and effective policy. It is encouraging to learn that almost all (96\%) schools were in compliance with the OTCA requirements and that the majority of nonsmoking students were in support of the ban. These findings demonstrate social support for tobacco-free schools and a readiness for legislation. Some students and school representatives thought the ban made it more difficult for non-smokers to be pressured into smoking and that students reduced the amount of smoking during the school day.

Challenges are inevitable with new policies. Almost all school administrators reported that a few students continued to smoke on school property. They were of the opinion that the ban did not discourage smoking. Extra work, due to additional demands on school personnel, was also reported in Ashley et al. as among the problems. Over time, continued efforts in education and enforcement from both Health Department and school staff should reinforce the policy and ultimately reduce the associated workload.

Unfortunately, in some settings students were reported at risk for traffic injury as a result of the increased time smokers spent on sidewalks or streets adjacent to the school. Obviously, a policy which is effective and efficient must be sensitive to local conditions. In Ottawa-Carleton, the Health Department has agreed to exemptions for smoking on school property when traffic placed students at risk. In keeping with the spirit of the law, these schools have to comply with a review process prior to allowing smoking back on school property. In the event of an exemption, the school would have to agree that the designated smoking area not be placed in a highly visible location or within 9 metres of an entrance way or window. In spite of 
difficulties, workable solutions do evolve with persistence and ongoing dialogue.

Unfortunately, complying with the Act doesn't always lead to the anticipated goal of decreased visibility or proximity of student smoking. In urban settings, the sidewalk a few metres from the school door is city property, and youth smoking then becomes a community issue whereby neighbours, retailers and other businesses are directly affected. While perceived as problems by school administrators, complaints from the community can heighten awareness and interest. School-based smoking prevention programs need to be enhanced and sustained by communitywide programs. We need everyone's help! The success of efforts to prevent and reduce tobacco use through schools relies on the awareness and commitment of parents and other members of the community.

\section{Next Steps}

It goes without saying that schools are the primary channel for tobacco prevention for children and youth. We must convince educators, parents and community members of the importance of tobacco prevention and work with them for the implementation of top quality educational programs. The decision students make about tobacco use will affect every aspect of their lives. In prepara- tion for life's journey, finishing school tobacco-free is important enough to warrant becoming a curriculum goal. Critical multiple interventions must include age-specific and continuously reinforced programs, teacher-training, support for cessation and ongoing program evaluation.

Students continue to receive contradictory messages about tobacco use. Why, if we prohibit youth under the age of 19 years to purchase tobacco and smoke on school property, do we permit them to possess tobacco at school? Total bans on under-aged smoking in public places are not yet the standard for regulation. As pointed out by the school representatives in the article by Northrup et al., students are told it is illegal to smoke on school property, however, they are able to smoke on public property "... whether that property is one mile or one inch away from school property."

Obviously gaps need to be filled so that the message on tobacco and the accompanying legislation are clear, consistent and constantly reinforced. Nonetheless, the OTCA represents a significant step forward and needs to be replicated in all provinces and territories. We must also continue to advocate for smoke-free municipal bylaws as well as support key initiatives at the federal level to curtail tobacco advertising, never forgetting that enforcement makes new legislation effective and ultimately determines public acceptance and high compliance. This is difficult. Enforcement is by its very nature confrontational and it casts us in an unpopular and uncomfortable role. But who said conquering tobacco would be a popularity contest for the faint of heart? Let's get on with the job of protecting the next generation, our children, from the tyranny of tobacco.

\section{REFERENCES}

1. Northrup DA, Ashley MJ, Ferrence R. The Ontario ban on smoking on school property: Perceived impact on smoking. Can J Public Health 1998;89(4):224-28.

2. Ashley MJ, Northrup DA, Ferrence R. The Ontario ban on smoking on school property: Issues and challenges in enforcement. Can J Public Health 1998;89(4):229-32.

3. Health Canada. Profile of youth aged 15-19. Survey on smoking in Canada, Cycle 1. Ottawa, 1994.

4. Ottawa-Carleton Department of Health. Tobacco use facts. Ottawa, 1998.

5. U.S. Department of Health and Human Services. Preventing tobacco use among young people: A report of the Surgeon General. Atlanta, GA: U.S. Department of Health and Human Services, Public Health Service, Centers for Disease Control and Prevention, National Center for Chronic Disease Prevention and Health Promotion, Office on Smoking and Health, 1994.

6. Health Canada. Smoking policies in schools. Ministry of Supply and Services Canada. Ottawa, 1995.

\section{Protéger nos enfants}

\section{Rob Cushman, MD, FRCPC, ${ }^{1}$ Katherine Robertson-Palmer, $R N, M S c N^{2}$}

À une époque où la santé publique insiste auprès des gouvernements pour que l'on protège nos enfants contre le tabac, la Revue canadienne de santé publique publie des informations utiles et actuelles sur les répercussions de l'OTCA (Ontario Tobacco Control Act, loi ontarienne sur le contrôle du tabac) dans les écoles. L'article

Service de la santé, Municipalité régionale d'OttawaCarleton

1. Médecin hygiéniste

2. Infirmière de santé publique, Programme de prévention du tabagisme, Division de la santé des adultes de Northrup et al. ${ }^{1}$ présente le point de vue des administrateurs scolaires au sujet de l'interdiction de fumer sur les lieux de l'école depuis l'adoption de l'OTCA. L'article d'Ashley et al. ${ }^{2}$ qui le complète présente les questions et les grands problèmes découlant de la mise en vigueur et de l'application de l'OTCA.

Nous sommes les témoins des avancées d'un programme de santé publique qui est passé successivement de l'éducation et de la promotion à sa traduction dans la loi pour aboutir à l'application de cette dernière. L'expérience nous montre que cette pro- gression est la seule qui permette de faire respecter la loi au niveau optimal, qu'il s'agisse des ceintures de sécurité, du tabac ou de n'importe quelle autre intervention.

Avant d'en venir au contrôle du tabac dans nos écoles, il est utile de passer en revue et d'actualiser ce que nous savons du tabac et des jeunes, afin de clairement définir le problème et l'importance de faire respecter la loi comme stratégie.

Au plan national, la prévalence du tabagisme chez les jeunes âgés de 15 à 19 ans s'établit à $29 \% .^{3}$ En raison principalement des réductions de la taxe fédérale intervenues en 1994, le tabagisme des adolescents connaît une augmentation alarmante. Dans l'est de l'Ontario, le taux de tabagisme chez les étudiants est passé de $22 \%$ en 1993 à 29 \% en 1997. ${ }^{4}$ La grande majorité des fumeurs commencent à fumer au cours des 
premières années de l'adolescence et les habitudes de tabagisme à l'âge adulte sont généralement déterminées entre 16 et 18 ans. ${ }^{3}$ À partir du moment où elle a été établie, l'accoutumance au tabac devient implacable. Les adolescents fumeurs reconnaissent leur accoutumance à la nicotine et veulent arrêter de fumer; en effet, plus de la moitié des adolescents qui fument à l'heure actuelle ont essayé de cesser de fumer pendant au moins une semaine. ${ }^{3}$ Toutefois, seulement 2 à $3 \%$ des adolescents, qui dans un premier temps ont essayé d'arrêter de fumer, y parviennent pendant une année ou deux. ${ }^{5}$ Les jeunes non fumeurs sont également vulnérables, que ce soit par exposition involontaire à une substance extrêmement toxique ou à cause des pressions qui s'exercent sur eux pour qu'ils se mettent à fumer.

C'est de toute évidence l'école qui est le terrain de combat où la guerre du tabac pour les prochaines générations sera gagnée ou perdue. C'est dans cet environnement, parmi leurs amis et leurs condisciples, que les adolescents prendront et enracineront la plus importante décision de leur vie s'agissant de leur santé. C'est la raison pour laquelle il est si important de passer en revue les politiques nationales en milieu scolaire et de tirer les leçons de l'expérience ontarienne découlant de l'adoption de l'OTCA.

Une analyse effectuée par Santé Canada en 1995 dans toutes les provinces a montré que presque toutes les écoles élémentaires et secondaires (97\%) disposaient d'une politique relative au tabagisme, bien que l'importance des restrictions imposées variait considérablement. ${ }^{6}$ Avant l'adoption de la loi ontarienne, l'application d'une politique anti-tabagisme dans les écoles fluctuait beaucoup entre les différentes municipalités de l'Ontario, ainsi qu'à l'intérieur de chacune d'entre elles. Ainsi, certaines écoles interdisaient à leurs étudiants de fumer à l'école, tandis que les enseignants et d'autres personnes étaient autorisés à fumer dans des zones désignées. Avec l'entrée en vigueur de l'OTCA, les écoles ont cessé d'envoyer un double message aux étudiants concernant le tabagisme.

\section{Ontario Tobacco Control Act, 1994}

La loi ontarienne (Ontario Tobacco Control Act, projet de loi 119) a été adop- tée en 1994 et, à l'époque, était considérée comme la législation la plus complète en Amérique du Nord. Les articles de la Loi portant sur les jeunes ont pour objectif de réduire leur tabagisme et de les protéger contre la fumée du tabac ambiante. Premièrement, la Loi restreint l'accès des jeunes aux produits du tabac en en limitant la vente et la fourniture aux jeunes de moins de 19 ans, en interdisant les distributeurs automatiques de paquets de cigarettes ainsi que la vente de "mini paquets» (contenant moins de 20 cigarettes) ou de cigarettes à l'unité. Deuxièmement, en vertu de l'article 9 de la Loi, fumer est interdit ou soumis à des restrictions à différents endroits, notamment dans les écoles telles qu'elles sont définies par la loi ontarienne sur l'éducation. L'interdiction frappe tous les bâtiments scolaires ainsi que tous les lieux de l'école en tout temps. Elle s'applique de façon uniforme au personnel, aux étudiants et aux personnes qui utilisent les lieux de l'école ou s'y rendent. On encourage les écoles à faire respecter cette interdiction lors des sorties et dans les bus scolaires au moyen d'une politique en ce sens.

L'enquête de Northrup et al. indique que l'OTCA est considérée comme une politique acceptable et efficace. Il est encourageant de voir que presque toutes les écoles (96\%) respectent les exigences de l'OTCA et que la majorité des étudiants non fumeurs sont en faveur de l'interdiction. Ces résultats montrent que la société est en faveur de l'interdiction de fumer dans les écoles et qu'elle est prête à accepter des mesures législatives en ce sens. Certains étudiants et représentants des écoles estiment que grâce à l'interdiction, il est devenu plus difficile d'exercer des pressions sur les non fumeurs pour qu'ils se mettent à fumer, et que les étudiants fumeurs ont diminué leur tabagisme au cours de la journée à l'école.

Toute nouvelle politique fait l'objet de contestation. Ainsi, la plupart des administrateurs scolaires ont déclaré que quelques étudiants continuaient de fumer sur les lieux de l'école et ont dit qu'à leur avis l'interdiction ne décourageait pas le tabagisme. Selon Ashley et al., les exigences supplémentaires imposées au personnel scolaire ont accru leur charge de travail entre autres problèmes. Avec le temps, les efforts soutenus d'éducation et d'application de la Loi par le service de santé et le personnel des écoles devraient renforcer la politique et, en fin de compte, diminuer la charge de travail qui lui est associée.

Malheureusement, à certains endroits, il apparaît que les étudiants fumeurs courent davantage de risques d'accident de la circulation parce qu'ils passent plus de temps sur les trottoirs ou les rues adjacentes à l'école. De toute évidence, pour être efficace et efficiente, une politique doit prendre en compte les conditions locales. Dans la région d'Ottawa-Carleton, le service de santé a accepté certaines dérogations à l'interdiction de fumer sur les lieux de l'école lorsque la circulation ambiante fait courir des risques aux étudiants. Afin de respecter l'esprit de la Loi, ces écoles doivent suivre une procédure d'examen de la situation avant d'autoriser de nouveau le tabagisme sur les lieux de l'école. Si une dérogation est accordée, l'école doit accepter que la zone désignée fumeur ne se situe pas dans un lieu très visible ou à moins de 9 mètres d'une entrée ou d'une fenêtre. En dépit des difficultés, le maintien du dialogue et la persévérance permettent de trouver des solutions applicables.

Malheureusement, respecter la Loi n'aboutit pas toujours à l'objectif visé, à savoir une moindre visibilité ou proximité du tabagisme des étudiants. En milieu urbain, les trottoirs qui se trouvent à quelques mètres des portes de l'école sont la propriété de la ville, et le tabagisme des jeunes devient alors une question communautaire qui concerne directement les voisins, les détaillants et autres commerces. Même si elles sont perçues par les administrateurs scolaires comme des problèmes, les plaintes émanant de la communauté sont de nature à accroître la sensibilisation et l'intérêt. Les programmes de prévention du tabagisme dans les écoles doivent être améliorés et renforcés par des programmes d'application généralisée à la communauté. Nous avons besoin de l'aide de tous! Le succès des efforts visant à prévenir ou à réduire la consommation de tabac dans les écoles dépend de la sensibilisation et de l'engagement des parents ainsi que des autres membres de la communauté.

...voir Éditorial, page 269 
agi avec diligence et efficacité. Il nous faut donc trouver des façons plus efficaces de dispenser ce type de programme par le personnel régulier des soins infirmiers auquel pourrait s'adjoindre le personnel des programmes de médecine de jour pour le suivi post-hospitalisation et l'intervention préhospitalisation s'il y a lieu.

Emmons et Goldstein ${ }^{10}$ décrivent l'hospitalisation comme une "window of opportunity " et un " teachable moment ", où la vulnérabilité aux dangers perçus du tabagisme, la motivation à cesser de fumer et la réceptivité à une intervention de cessation sont à un point culminant. Les études réalisées à ce jour se révèlent suffisamment concluantes pour que les expérimentations futures portent non pas sur la pertinence d'implanter des programmes de cessation du tabagisme auprès des patients hospita-lisés mais plutôt sur : les meilleures façons d'augmenter la fidélité du personnel au programme, la pertinence d'utiliser du personnel spécifiquement chargé de cette tâche, la place des interventions pré-hospitalières et l'intensité re-quise des interventions après l'hospitalisation.

\section{BIBLIOGRAPHIE}

1. Boyle P. Cancer, cigarette smoking and premature death in Europe: A review including the recommendations of European Cancer Experts Consensus Meeting, Helsinki, October 1996. Lung Cancer 1997;17(1):1-60.

2. Bellerose C, Lavallée C, Camirand J. Enquête sociale et de santé 1992-1993. Faits saillants. Gouvernement du Québec. Santé-Québec. p.18.

3. Glasgow RE, Stevens VJ, Vogt TM, et al. Changes in smoking associated with hospitalization: Quit rates, predictive variables and intervention implications. Am J Health Prom 1991;6(1):24-29.

4. Simon JA, Browner WS, Mangano DT Predictors of smoking relapse after noncardiac surgery. Am J Public Health 1992;82(9):1235-37.
5. Taylor CB, Houston-Miller N, Killen JD, DeBusk RF. Smoking cessation after acute myocardial infarction: Effects of a nurse-managed intervention. Ann Intern Med 1990;113(2):118-23.

6. Bandura A. Social foundations of thought and action: A social cognitive theory. In: Santé et Société: collection promotion de la santé. MSSS, 1988; 10-12.

7. Wewers ME, Bowen JM, Stanislaw AE, Desimone VB. A nurse-delivered smoking cessation intervention among hospitalized postoperative patients - influence of a smoking-related diagnosis: A pilot study. Heart \& Lung 1994;23(2):151-56.

8. Orleans CT, Kristeller JL, Gritz ER. Helping hospitalized smokers quit: New directions for treatment and research. J Consult Clin Psychol 1993;61(5):778-89.

9. Miller NH, Smith PM, DeBusk RF, et al. Smoking cessation in hospitalized patients. Arch Intern Med 1993;157:409-12.

10. Emmons KM, Goldstein MG. Smokers who are hospitalized: A window of opportunity for cessation interventions. Prev Med 1992;21:262-69.

Reçu : 23 juin 1997

Accepté : 30 janvier 1998
Éditorial, suite de la page 223

\section{Prochaines étapes}

Il va sans dire que les écoles sont le principal endroit où prévenir le tabagisme des enfants et des jeunes. Nous devons convaincre les éducateurs, les parents et les membres de la communauté de l'importance de la prévention du tabagisme, et collaborer avec eux pour mettre en oeuvre des programmes éducatifs de toute première qualité. Le choix que font les étudiants vis à vis du tabagisme aura des répercussions sur tous les aspects de leur vie. Par rapport à la vie qui les attend, finir leurs études sans être fumeur est suffisamment important pour justifier qu'on en fasse un objectif du programme scolaire. Les nombreuses interventions nécessaires doivent comprendre des programmes de renforcement continu adaptés à la catégorie d'âge, la formation des enseignants, l'appui à la cessation du tabagisme et l'évaluation régulière des programmes mis en oeuvre.

Les étudiants continuent de recevoir des messages contradictoires au sujet du tabagisme. Ainsi, pourquoi si nous interdisons aux jeunes de moins de 19 ans d'acheter du tabac et de fumer sur les lieux de l'école, leur permettons-nous d'avoir en leur possession du tabac à l'école? L'interdiction totale de fumer dans les lieux publics pour les personnes qui n'ont pas l'âge ne constitue toujours pas la norme réglementaire. Comme le font remarquer les représentants des écoles dans l'article de Northrup et al., d'une part on dit aux étudiants qu'il est illégal de fumer sur les lieux de l'école, et d'autre part on ne les empêche pas de fumer dans les lieux publics «...que ces lieux soient éloignés ou au contraire à proximité de l'école». ${ }^{1}$

De toute évidence, il y a des lacunes à combler pour que le message anti-tabac et la législation d'accompagnement soient bien clairs, cohérents et renforcés en permanence. Quoi qu'il en soit, l'OTCA est un pas important dans la bonne direction qui doit être fait dans toutes les provinces et les territoires. Nous devons également continuer de revendiquer des règlements municipaux interdisant le tabagisme et soutenir les grandes initiatives lancées par le gouvernement fédéral pour limiter la publicité du tabac, sans jamais oublier que ce sont les mesures d'application de la loi qui la rendent efficace et qui, en fin de compte, déterminent son acceptation et son respect généralisés par le public. Ce n'est pas chose facile. Faire appliquer une loi est par nature un processus antagoniste qui nous fait jouer un rôle impopulaire et souvent inconfortable. Mais qui a jamais prétendu que se battre contre le tabac était un concours de popularité pour les moins vaillants? Mettons-nous à la tâche et continuons le combat pour protéger la prochaine génération, nos enfants, contre la tyranie du tabac.

\section{BIBLIOGRAPHIE}

1. Northrup DA, Ashley MJ, Ferrence R. The Ontario ban on smoking on school property: Perceived impact on smoking. Revue can. de sante publique 1998;89(4):224-28.

2. Ashley MJ, Northrup DA, Ferrence R. The Ontario ban on smoking on school property: Issues and challenges in enforcement. Revue can. de santé publique 1998;89(4):229-32.

3. Santé Canada. Profile of youth aged 15-19. Survey on smoking in Canada, Cycle 1. Ottawa, 1994.

4. Ottawa-Carleton Department of Health. Tobacco use facts. Ottawa, 1998.

5. U.S. Department of Health and Human Services. Preventing tobacco use among young people: A report of the Surgeon General. Atlanta, GA: U.S. Department of Health and Human Services, Public Health Service, Centers for Disease Control and Prevention, National Center for Chronic Disease Prevention and Health Promotion, Office on Smoking and Health, 1994.

6. Santé Canada. Smoking policies in schools. Ministère des Approvisionnements et Services Canada. Ottawa, 1995. 